\title{
FUZZY LOGIC APPLICATIONS IN HORTICULTURE AND A SAMPLE DESIGN FOR JUICE VOLUME PREDICTION IN POMEGRANATE (PUNICA GRANATUM L.)
}

\author{
PAKYÜREK, M. ${ }^{1}-$ AYDIN, Y. $^{2}-$ MIKAIL, N. ${ }^{3 *}$ \\ ${ }^{I}$ Department of Horticulture, Faculty of Agriculture, Siirt University, 56100, Siirt, Turkey \\ ${ }^{2}$ Depart of Biosystem Engineering, Faculty of Agriculture, Siirt University, 56100, Siirt, Turkey \\ ${ }^{3}$ Department of Animal Science, Faculty of Agriculture, Siirt University, 56100, Siirt, Turkey \\ *Corresponding author \\ e-mail: naziremikail@siirt.edu.tr \\ (Received $19^{\text {th }}$ Oct 2018; accepted $28^{\text {th }}$ Jan 2019)
}

\begin{abstract}
Fuzzy expert systems search for a solution based on the expertise of people who are experts in a particular field. This could be described as a kind of advisory system edited on computer. The use of natural language on the basis of fuzzy logic and easier understanding of system logs provide this technique to resolve many daily and current problems. In this study, a sample expert system to estimate juice volume in pomegranate was designed, using the fuzzy logic method, which closest to the logic of the human mindset. Recording of data was performed on the private farm of the province of Siirt, Turkey. The Fuzzy Logic Interface of MATLAB Program was used in the designing phase of the system. The evaluation of the model was carried out according to coefficient of determination and coefficient of correlation. The model revealed $\mathrm{R}^{2}=80 \%$ coefficient of determination, and $\mathrm{r}=0.89$ coefficient of correlation. With more informative parameters, the error rate can be decreased. Fuzzy logic seems one of the useful tools with prediction purposes in horticulture.
\end{abstract}

Keywords: fuzzy expert system, Punica granatum L. cv. Zivzik, juice volume, fruit size, irrigation

\section{Introduction}

Pomegranate (Punica granatum L.) is a tropical and subtropical fruit species, in the cultivation of which India, Iran and China are the top ranking in the world, followed by Turkey and USA. The annual production of pomegranate cultivation is over 100,000 tons in Turkey, which is available in 48 provinces of the country. Pomegranate is a perennial and drought-tolerant plant. Arid and semiarid zones are popular for growing pomegranate trees (Asgary et al., 2014). The flowering and fruit setting period varies according to low or high altitude conditions. This particularly affects the early or late opening of flowers. It is indicated that the colors and sizes are not normal due to the inadequate temperatures (Onur, 1988; El-Sese, 1988). Pomegranate juice is a good source of fructose, sucrose, and glucose. It also has some of the simple organic acids such as ascorbic acid, citric acid, fumaric acid, and malic acid (Asgary et al., 2014). Morever it is a polyphenol-rich fruit juice with a high antioxidant capacity. This fruit can help preventing or treating several cardiovascular risk factors including hypertension, hypercholesterolemia, oxidative stress, hyperglycemia, and inflammation (Chong et al., 2010; Wang et al., 2014; Sahebkar et al., 2017).

Agricultural production is a complex system requiring knowledge and information from many diverse sources. Agricultural specialist assistance is not always available for the farmer who needs it. In order to support the farmer expert systems were identified as 
a powerful tool with extensive potential in agriculture (Prasad and Vinaya Babu, 2006; Dath and Balakrishnan, 2013; Kolhe and Gupta, 2014).

During the last five decades, the potential of electronic data processing has been used to an increasing degree to support human decision making in different ways. Since the late 1970s and early 1980s decision support systems found their way into management and engineering. Evaluation, diagnosis, prediction could be classified as decision support systems. Even though fuzzy set theory can be used in all three "prototypes" we shall concentrate on "expert systems" only because the need and problem of managing uncertainty of many kinds is most apparent there; hence, the application of fuzzy set theory is most promising and advanced. In operations research the modeling of problems is normally being done by the OR-specialist. The user then provides input data and the mathematical model provides the solution to the problem by means of algorithms selected by the OR-specialist (Zimmermann, 2001). While the typical ORmodel or software package normally supports the expert, an expert system is supposed to model an expert and make his expert knowledge available to non-experts for purposes of decision making, consulting, diagnosis, learning, or research (Konopasek and Jayaraman, 1984).

The most relevant approaches of fuzzy expert system (FES) in horticulture is fruit sorting and grading system (May and Amaran, 2011; Nandi et al., 2012; Razak et al., 2012; Teoh et al., 2013; Hasan et al., 2014; Suksawat and Komkum, 2015; Nandi et al., 2016); fruit counting (Kumar et al., 2017; Qureshi et al., 2017), plant watering management (Ge et al., 2013; Ying et al., 2017), plant yield prediction (Srinivasan et al., 2009; Papageorgiou et al., 2013). Brotons et al. (2017) in their study tried to find relation between pomegranate maturity index with to solar net radiation by means of fuzzy approach. As a result of the study, they reported the possibility of prediction the most likely date from which the fruit will be ready for harvest, for a given latitude.

The main purpose of this study was to introduce fuzzy expert systems in estimating the juice volume without requiring any laboratory work by using the pomegranate features such as different harvest time, irrigation amounts, and fruit size.

\section{Materials and methods}

\section{Plant material}

The plant material of the study is pomegranate (Punica granatum L.) cv. Zivzik, obtained from an 8 year-old orchard located at $37^{\circ} 57^{\prime} 17^{\prime \prime}$ Northern and $41^{\circ} 51^{\prime} 07^{\prime \prime}$ Eastern coordinates, in Kezer region of the Central district of Siirt, Turkey (Fig. 1). Experiment was settled with 3 replications according to the design of random blocks.

The irrigation in pomegranate cultivation is one of the important parameters and therefore we have considered it as one of the input parameters. The amount of irrigation water was designed based on the fact that $50 \%, 75 \%$ and $100 \%$ of the open water surface evaporation values, obtained from the experimental area, were applied as irrigation water when they reached $80 \mathrm{~mm}$ and $120 \mathrm{~mm}$ (Aydin et al., 2017). The amounts of irrigation water applied to the treatments were between $292.6 \mathrm{~mm} \mathrm{(50 \% ),}$ $438.8 \mathrm{~mm}(75 \%)$ and $585.2 \mathrm{~mm}(100 \%)$. In the experiment, during the 4 weeks from the middle of October to the beginning of November, five fruit samples from each Zivzik pomegranate tree were taken weekly and their physical and chemical properties were examined. 


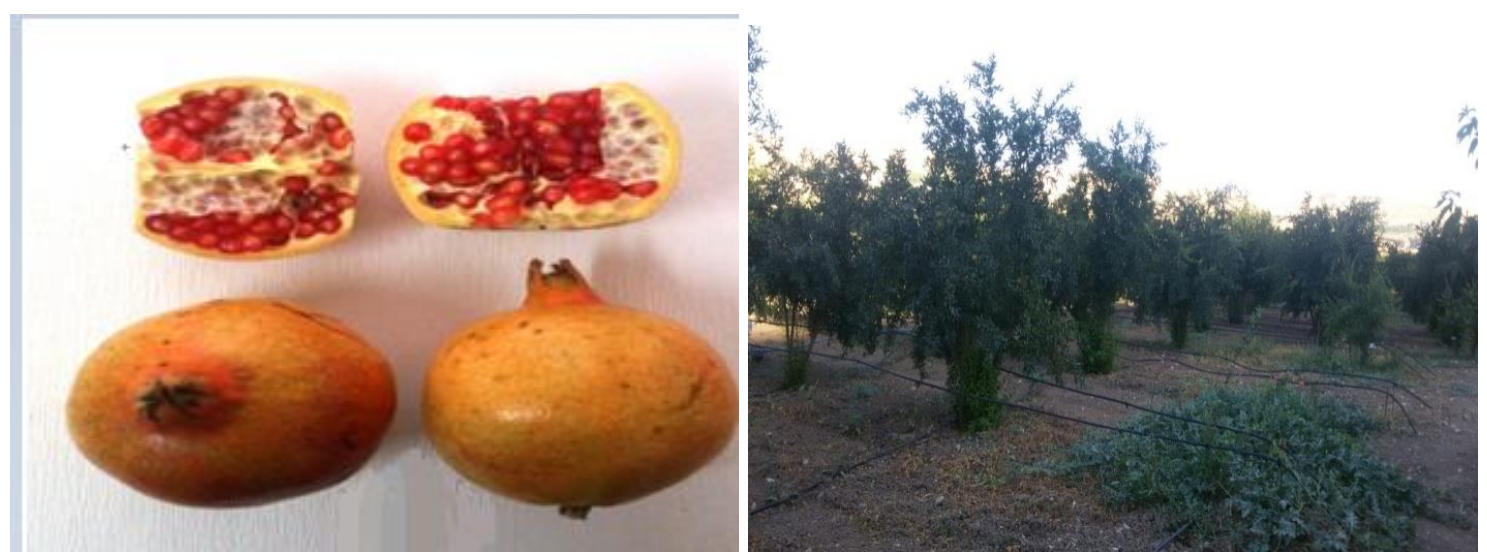

Figure 1. Zivzik variety of pomegranate: fruit and orchard

Much of human knowledge is a collection of rules and facts which, for the most part are neither totally certain, nor totally consistent, the storage of these vague and uncertain portions of knowledge by using fuzzy sets seems much more appropriate than the use of crisp concepts and symbolism, "management of uncertainty" of human thinking, when modeled in expert systems, might also increase efficiency, that is, decrease answering time (Zadeh, 1983; Zimmermann, 2001).

The fruit samples used were harvested respectively on October 16, October 23, October 31, and November 6, 2015. The fruits were determined in terms of fruit weight, fruit length, fruit width, aril yield (\%), fruit juice ratio, total soluble solids $(\%)$, titrable acidity (\%), TSS / TA and aril color. Pomological analyzes of these samples were carried out in the laboratory of Siirt University. Fruit size (FS) trait from the analyzed pomological characteristics was used in the prediction.

\section{Fuzzy logic based expert system}

An expert system is a computer program that solves problems that heretofore required significant human expertise by using explicitly represented domain knowledge and computational decision procedures (Kastner and Hong, 1984). The general structure of an expert system is shown in Figure 2. The Knowledge Acquisition Module supports the building of an expert system's knowledge base. The subject of knowledge acquisition for knowledge-based systems falls conveniently into two parts depending on whether the knowledge is elicited from the experts by knowledge engineers or whether that knowledge is acquired automatically by the computer using some form of automatic learning strategy and algorithms (Graham and Jones, 1988; Zimmermann, 2001).

General structure of FES for juice volume prediction in pomegranate is given in Figure 3.

Input and output values and their intervals in established model for the prediction of fruit juice volume (FJL) by means of FL are shown in Table 1. As the table shows, harvesting period (HP), amount of irrigation water (AIW) and FS were used as input data, and the output data of fruit juice volume were obtained.

Verbal expressions and fuzzy sets for HP, AIW, FS and FJV are given in Table 2. As Table 2 shows, the verbal expressions established for HP were $1^{\text {st }}$ period, $2^{\text {nd }}$ period, $3^{\text {rd }}$ 
period and $4^{\text {th }}$ period; for AIW deficit, medium and enough; for FS very small, small, normal, large and very large; and for FJV very low, low, normal, high and very high.

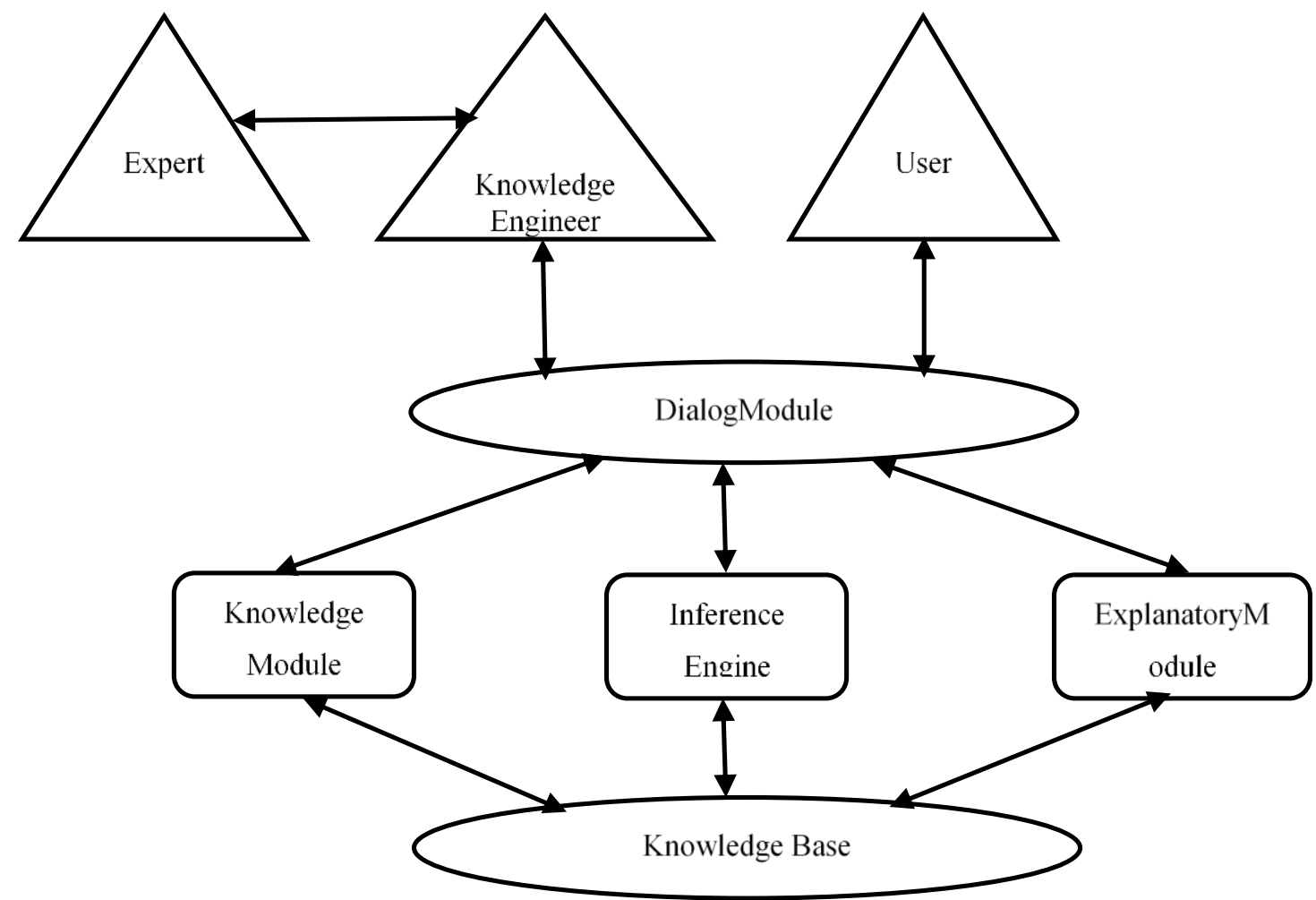

Figure 2. Structure of an expert system

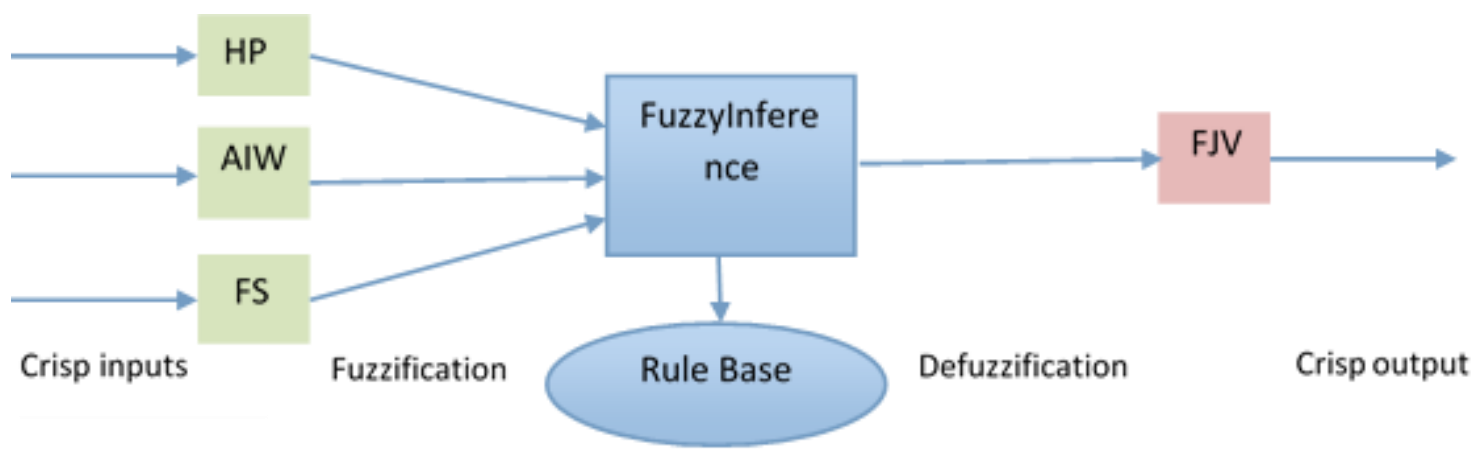

Figure 3. Scheme of the developed FES

Table 1. Input-output values and their intervals

\begin{tabular}{c|c|c}
\hline Input/Output & Trait & Intervals \\
\hline \multirow{3}{*}{ Input data } & HP & $0-5$ (integer) \\
& AIW & $0-4$ (integer) \\
& FS & $76-270(\mathrm{gr})$ \\
\hline Output data & FJV & $13-73(\mathrm{ml})$ \\
\hline
\end{tabular}


Table 2. Verbal expressions for HP, AIW, FS and FJV

\begin{tabular}{c|c|c}
\hline Trait & Verbal expression & Value intervals \\
\hline \multirow{4}{*}{ HP } & $1^{\text {st }}$ period & $0<x<2$ \\
& $2^{\text {nd }}$ period & $1<x<3$ \\
& $3^{\text {rd }}$ period & $2<x<4$ \\
& $4^{\text {th }}$ period & $3<x<5$ \\
\hline \multirow{3}{*}{ AIW } & Deficit & $0<x<2$ \\
& Medium & $1<x<3$ \\
& Enough & $2<x \leq 4$ \\
\hline \multirow{3}{*}{ FS } & Very small & $76 \leq x<110$ \\
& Small & $100<x<180$ \\
& Normal & $170<x<200$ \\
& Large & $190<x<220$ \\
& Very large & $210<x \leq 270$ \\
\hline \multirow{3}{*}{ FJV } & Very low & $13 \leq x<23$ \\
& Low & $20<x<38$ \\
& Normal & $37<x<47$ \\
& High & $45<x<53$ \\
& Very high & $52<x \leq 73$ \\
\hline
\end{tabular}

Various functions were used to graph input and output membership. Here, triangular (Eq. 1) and trapezoidal (Eq. 2) membership functions were used to calculate membership degrees of the input and output values generated (Baykal, 2004).

$$
\begin{gathered}
\mu_{A}\left(x ; a_{1}, a_{2}, a_{3}\right)=\left\{\begin{array}{ccc}
a_{1} \leq x \leq a_{2} & \text { then } & \left(x-a_{1}\right) /\left(a_{2}-a_{1}\right) \\
a_{2} \leq x \leq a_{3} & \text { then } & \left(a_{3}-x\right) /\left(a_{3}-a_{2}\right) \\
x>a_{3} \text { veya } x<a_{1} & \text { then } & 0
\end{array}\right. \\
\mu_{A}\left(x ; a_{1}, a_{2}, a_{3}, a_{4}\right)=\left\{\begin{array}{ccc}
a_{1} \leq x \leq a_{2} & \text { then } & \left(x-a_{1}\right) /\left(a_{2}-a_{1}\right) \\
a_{2} \leq x \leq a_{3} & \text { then } & 1 \\
a_{3} \leq x \leq a_{4} & \text { then } & \left(a_{4}-x\right) /\left(a_{4}-a_{3}\right) \\
x>a_{4} \text { veya } \mathrm{x}<\mathrm{a}_{1} & \text { then } & 0
\end{array}\right.
\end{gathered}
$$

Mamdani inference technique was used as fuzzy inference method (Mamdani and Assilian, 1975). A centroid technique method (Cox, 1999) was used for defuzzification of fuzzy data. It finds the point where a vertical line would slice the aggregate set into two equal masses. Mathematically this centre of gravity (COG) can be expressed as: 


$$
\operatorname{COG}=\frac{\int_{a}^{b} \mu_{A}(x) x d x}{\int_{a}^{b} \mu_{A}(x) d x}
$$

A centroid defuzzification method $(E q .3)$ finds a point representing the centre of gravity of the fuzzy set A, on the interval ab.

\section{Evaluation}

In this study, coefficient of correlation and coefficient of determination (Eq.4) (Spiegel et al., 2009) between real estimated and predicted by means of FES were calculated in term of accuracy and efficiency of the system.

$$
R^{2}=\frac{\sum_{i=1}^{n}\left(\hat{Y}_{i}-\bar{Y}\right)^{2}}{\sum_{i=1}^{n}\left(Y_{i}-\bar{Y}\right)^{2}}
$$

where: $Y_{\bar{i}}$ - observed value, $\left(\widehat{Y}_{i}\right)$ - predicted value, $\bar{Y}$ - arithmetic mean, $\mathrm{n}-$ the total number of observations.

\section{Results and discussion}

As a result of analysis of variance, it was determined that there was a significant difference between harvesting periods according to juice volume $(\mathrm{p}<0.05)$. A significant positive correlation was found between fruit weight and fruit juice volume $(\mathrm{p}<0.01)$.

Descriptive analysis of the data revealed that the average fruit juice volume (FJV) of pomegranate in $4 \mathrm{HP}$ and 3 different irrigation amount (deficit, medium, enough) varied from $18.39 \pm 2.73$ to $51.33 \pm 9.20 \mathrm{ml}$; fruit weight of pomegranate (FS) from $91.18 \pm 10.80$ to $226.52 \pm 25.80 \mathrm{~g}$ respectively.

For the FL realized via the MATLAB program (Mathworks, 2009), 3 inputs (HP, AIW, FS) and 1 output (FJV) were available. The HP graph formed in MATLAB, as illustrated in Figure 4, uses the four harvesting period as 4 the conditional fuzzy sets. In FES, however, this input is entered as discrete. The AIW graph formed in MATLAB and containing 3 verbal expressions is illustrated in Figure 5. It consists of 3 verbal variables. Since the amount of irrigation water generally changes according to the number of factors such as the amount of irrigation made by the farmer, the amount of rainfall, the amount of evaporation associated with the seasonal temperature, and the soil structure. Just farmer's decision concerning plant irrigation basically can be used. We used this input as the crisp input in our model. For deficit irrigation 1 will be entered, 2 for medium, 3 for enough irrigation. In the FS graph, illustrated in Figure 6, for the very small and very large fuzzy sets the trapezoidal membership functions was selected, and for the small, medium and large fuzzy set the triangular function was used. Figure 7 shows the output graphic FJV. The FJV graph, divides the output data into five ranges as very low, low, normal, high and very high, and shows the verbal expression memberships. For the very low and very high fuzzy sets the trapezoidal membership function was selected; for the normal and high fuzzy sets the triangular function was used.

Rule base of the FES was contained of 25 rules. Rules were formed from the data (HP, AIW, FS) and taking into account the FJV as measured in the laboratory. Table 3 shows some rules given in our FES. 
Table 3. Some rules from developed rulebase

\begin{tabular}{l|l|l|l|l|l|l|l|c}
\hline Rule 1: & If HP is & ' 1 st Period' & AIW is & 'Medium' & FS is & 'Small' & Then FJV is & 'Normal' \\
Rule 2: & If HP is & ' $2^{\text {nd }}$ Period' & AIW is & 'Medium' & FS is & 'Normal' & Then FJV is & 'Low' \\
Rule 3: & If HP is & ' $3^{\text {rd }}$ Period' & AIW is & 'Enough' & FS is & 'Very large' & Then FJV is & 'Very high' \\
Rule 4: & If HP is & '4 $4^{\text {th }}$ Period' & AIW is & 'Enough' & FS is & 'Very small' & Then FJV is & 'Very low' \\
Rule 5: & If HP is & ' $2^{\text {nd }}$ Period' & AIW is & 'Deficit' & FS is & 'Normal' & Then FJV is & 'Normal' \\
\hline
\end{tabular}

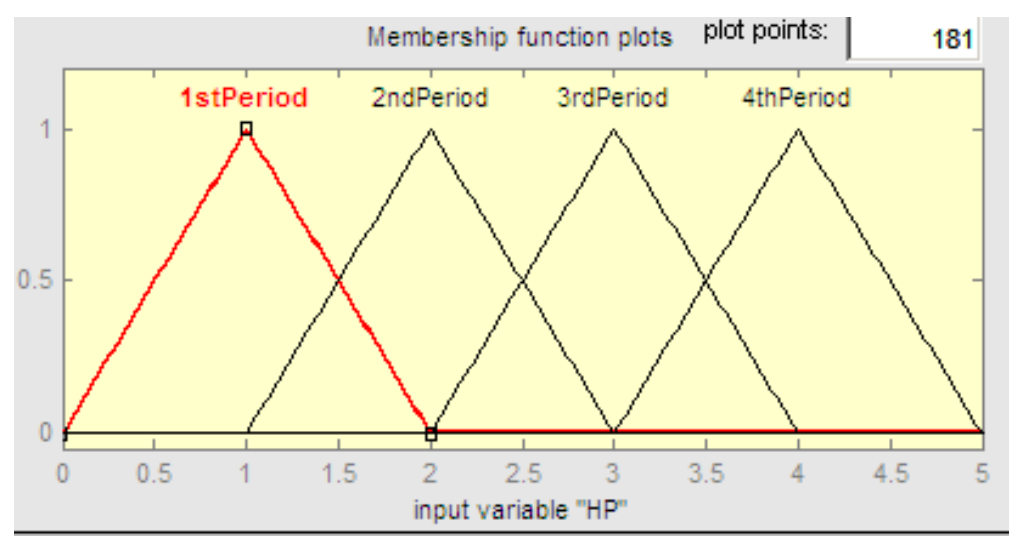

Figure 4. HP membership function graph

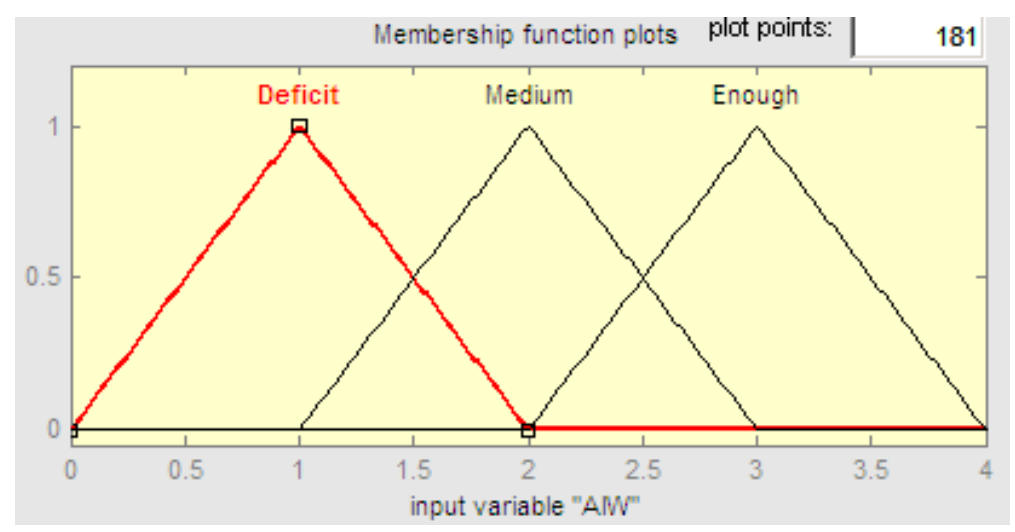

Figure 5. AIW membership function graph

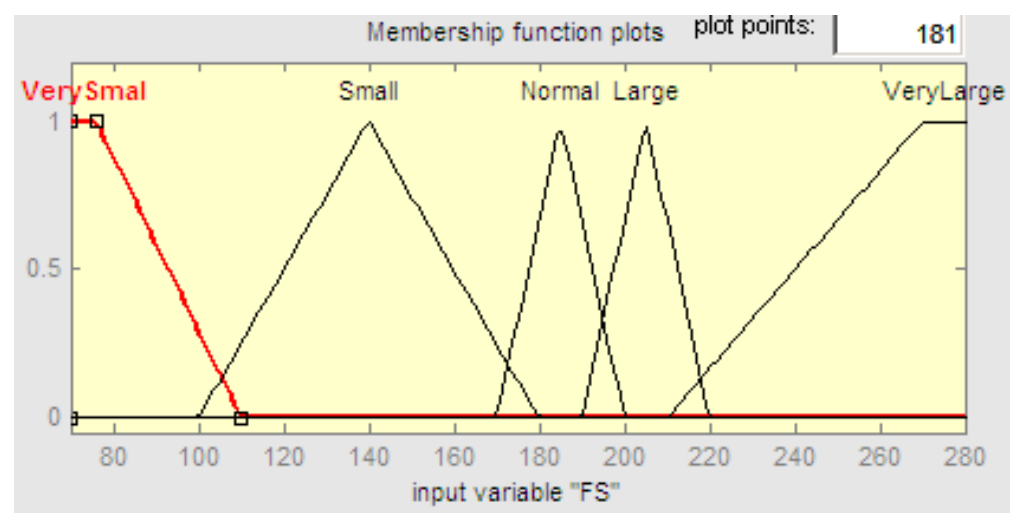

Figure 6. FS membership function graph 


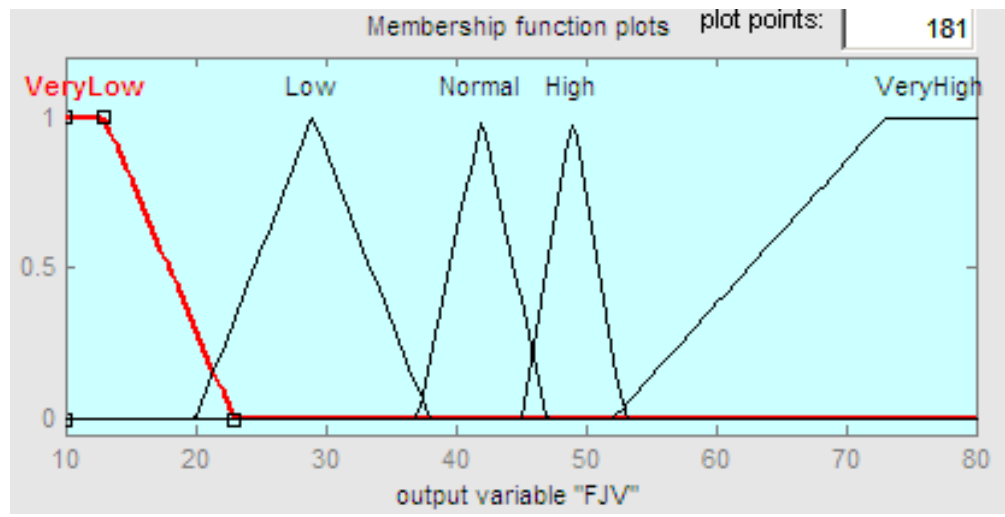

Figure 7. FJV membership function graph

Figures 8 and 9 show the 3-dimensional relationships between the HP, AIW, FS and FS.

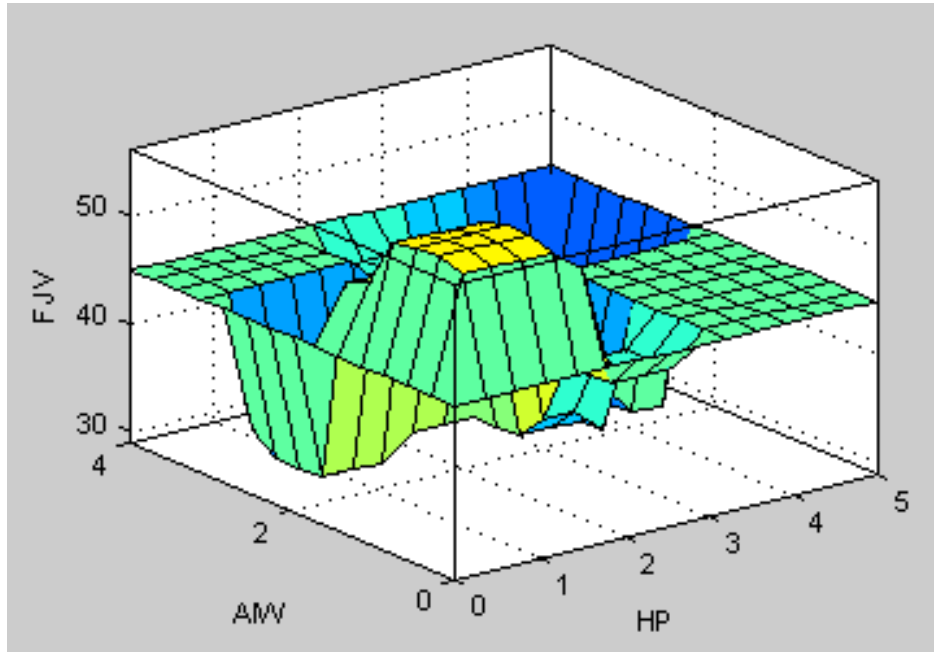

Figure 8. 3-D relation between AIW, HP and FJV

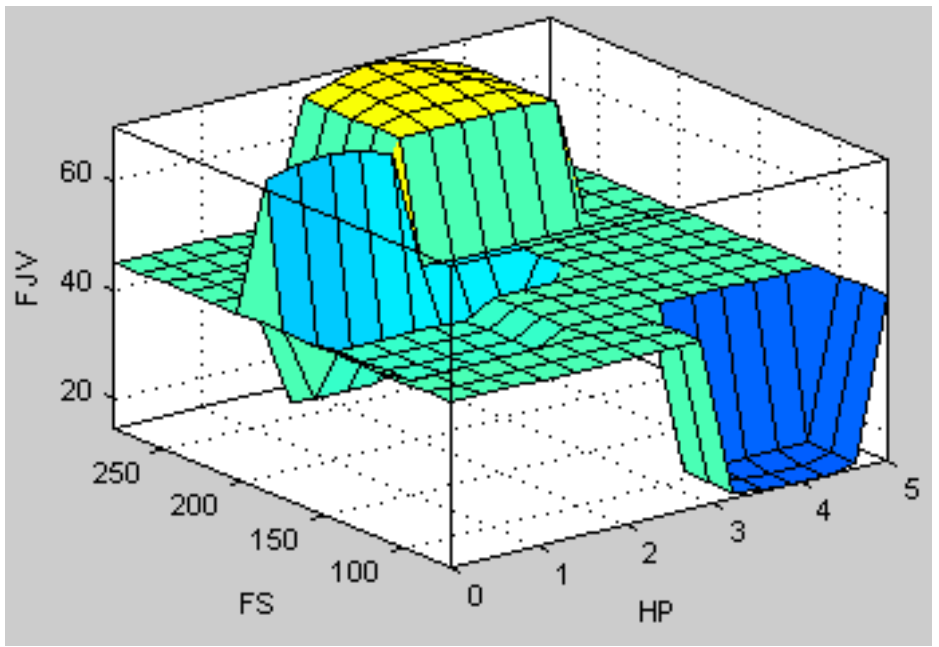

Figure 9. Relation between FS, HP and FJV 
For example, given $1^{\text {st }}$ Period for HP, 2-medium for AIW, and $165 \mathrm{mg}$ for FS, the observed output value appears next to the statement $42 \mathrm{ml}$ for FJV (Fig. 10a). Other results can be found by supplying different input data, for example, given $\mathrm{HP}=3^{\text {rd }}$ Period, AIW=enough, and FS $=250 \mathrm{mg}$, the appropriate response for these inputs is FJV $=69.3 \mathrm{ml}$ (Fig. 10b).
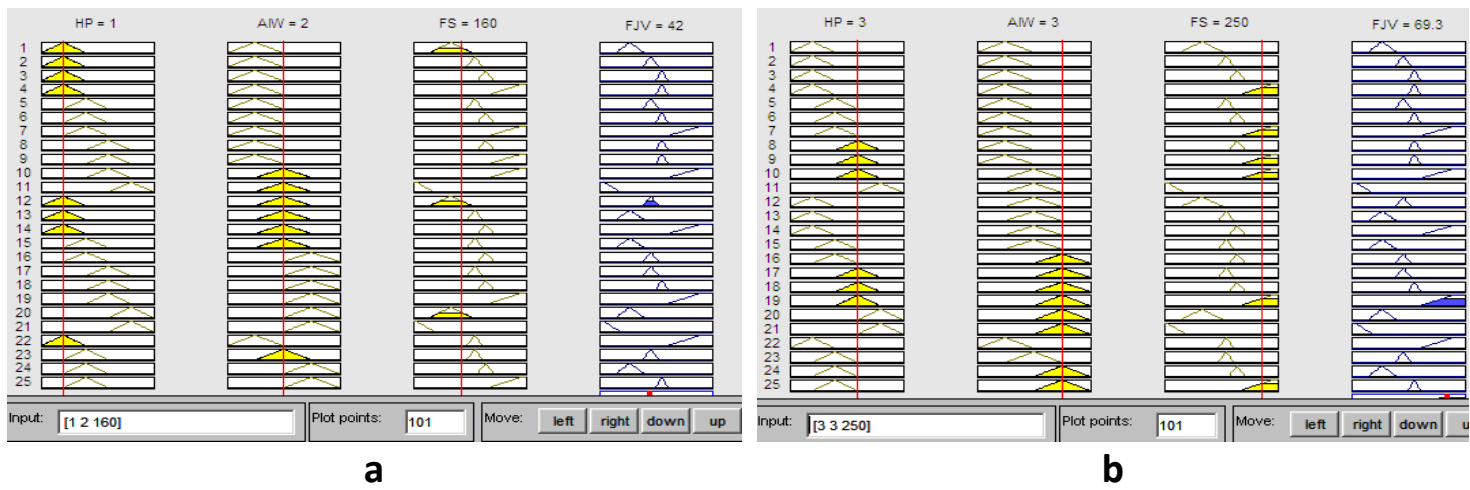

Figure 10. Simulation results for the given inputs

As shown in Figure $10 a$ and $b$, different rules were fired for the different inputs. Thereafter, all data were run through the FL model, with the results of FJV. The coefficient of correlation between real estimated and predicted by means of FL was 0.89 (Fig. 11).

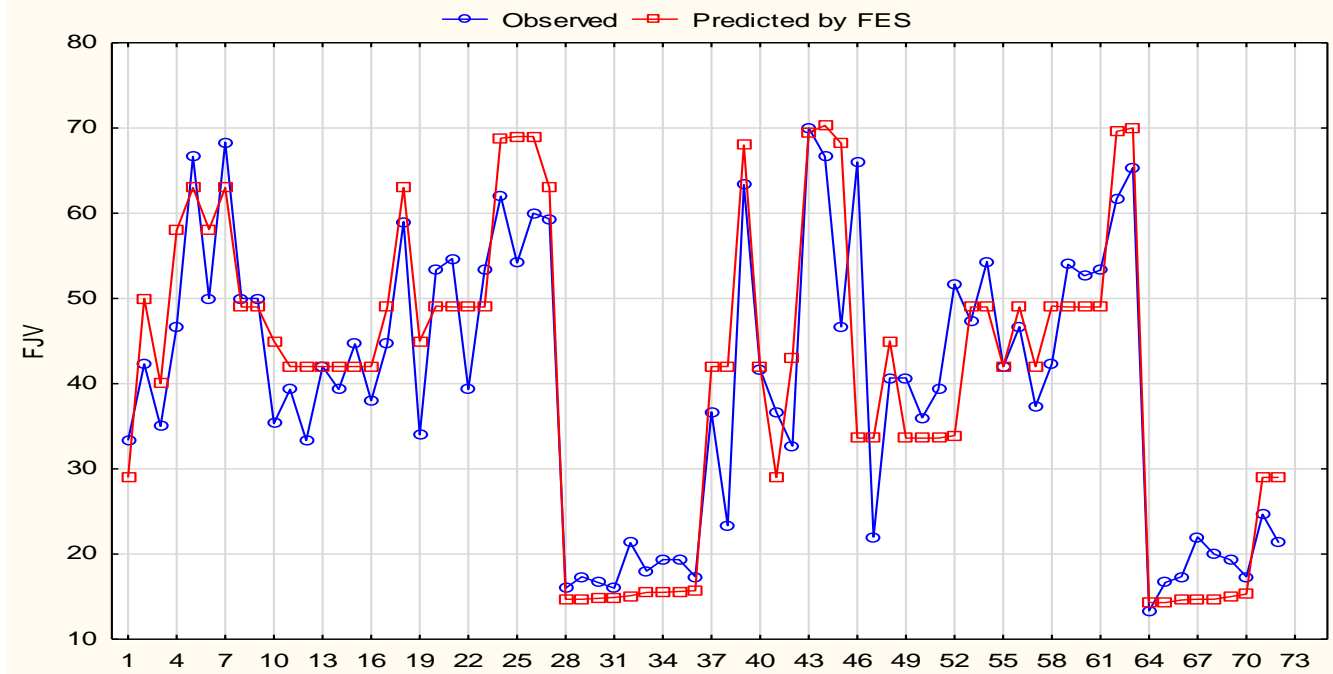

Figure 11. Relation between FJV measured in laboratory and predicted via FES

The coefficient of determination was found to be $80 \%$. With the aim of fast, but accurate and easy way of finding volume, weight, and surface area of a crop in the study of Dorvlo et al. (2012) the prediction equation for weight can be used with an appreciable coefficient of determination of $68.89 \%$. Prediction accuracy of the current study was found higher than the study of Dorvlo et al. (2012). 


\section{Conclusion}

This paper assayed pomegranate juice volume estimation by means of the FL. Using the input data of HP, AIW and FS model was able to predict fruit juice volume at a level of success. In the end, the study's FL model revealed coefficient of determination rate of $80 \%$. It can be said that a fuzzy expert system can be applied in areas where mathematical modeling is difficult. In addition, the establishment of such expert systems in agriculture using fuzzy logic will help real-time, appropriate and rapid decision-making in land conditions, taking advantage of analytical decision-making capabilities of experts. In the model we build, we can develop an expert system, which gives more definite results in future by further developing and fuzzifiing the other inputs as much as possible, that can guide the producers of pomegranate breeding. Given these results, it is recommended that a prediction system be devised by adding more improved FL model with high sensitivity. Such a system will eliminate the need for intensive laboratory work in lieu of data obtained from farm. Considering all the factors, a more punctual and efficient system can be developed in the future.

\section{REFERENCES}

[1] Asgary, S., Javanmard, S., Zarfeshany, A. (2014): Potent health effects of pomegranate. Advanced Biomedical Research 3: 100.

[2] Aydın, Y., Mikail, N., Pakyürek, M., Saltuk, B., Seven, M. (2017): Water-yield relationship of zivzik pomegranate under deficit irrigation conditions. - Scientific Papers Series E. Land Reclamation, Earth Observation \& Surveying, Environmental Engineering 6: 81-86.

[3] Baykal, N., Beyan, T. (2004): Bulanık mantık: ilke ve temelleri. - Bıcaklar Kitabevi, Ankara.

[4] Brotons, J. M., Legua, P., Melgarejo, P., Manera, F. J., Hernández, F., Martínez, J. J. (2017): A fuzzy approach for relating a pomegranate maturity index with to solar net radiation. - Journal of Agricultural Science and Technology 19(3): 669-680.

[5] Chong, M. F. F., Macdonald, R., Lovegrove, J. A. (2010): Fruit polyphenols and CVD risk: a review of human intervention studies. - The British Journal of Nutrition 104(3): 28-39.

[6] Cox, E. (1999): The Fuzzy Systems: A Practitioner's Guide to Building, Using, and Maintaining Fuzzy Systems. $2^{\text {nd }}$ ed. - Academic Press, San Diego, CA.

[7] Dath, A., Balakrishnan, M. (2013): Development of an expert system for agricultural commodities. - The International Journal of Computer Science and Applications (TIJCSA) 2(7): 74-90.

[8] Dorvlo, S. Y., Bani, R. J., Sinayobe, E. (2012): Prediction of volume, weight and surface area of banana (Musa Acuminata) using picture image analysis. - Journal of Food, Agriculture and Environment 10(3\&4): 112-114.

[9] El-Sese, A. M. (1988): Effect of time of fruit setting on the quality of some pomegranate cultivars. - Agric. Sci. 19(3):55-69.

[10] Ge, Z., Zhang, R., Yu, Y., Wu, W. (2013): An automatic plant irrigation system based on fuzzy control. - Applied Mechanics and Materials 303-306: 1162-1166.

[11] Graham, I., Jones, P. L. (1988): Expert Systems: Knowledge, Uncertainty and Decision. Chapman \& Hall, London, New York.

[12] Hasan, S. A., Kin, T. Y., Sauddinsa Duddin, S., Aziz, A. A., Othman, M., Mansor, A. R., Parnabas, V. (2014): Automated mango fruit assessment using fuzzy logic approach. AIP Conference Proceedings 1602: 335-343.

[13] Kastner, J. K., Hong, S. J. (1984): A review of expert systems. - EJOR 18: 285-292. 
[14] Kolhe, S., Gupta, G. K. (2014): Intelligent systems for agriculture domain. - International Journal of Computer Applications 0975-8887: 14-18.

[15] Konopasek, M., Jayaraman, S. (1984): Expert systems for personal computers. - Byte 137-154.

[16] Kumar, P. S., Panda, S., Kumari, S. B., Kumar, A. R. (2017): On tree detection, counting \&amp; post- harvest grading of fruits based on image processing and machine learning approach-a review. - International Journal of Engineering and Technology (IJET). http://doi.org/10.21817/ijet/2017/v9i2/170902058.

[17] Mamdani, E. H., Assilian, S. (1975): An experiment in linguistic synthesis with a fuzzy logic controller. - International Journal of Man-Machine Studies 7(1): 1-13.

[18] Mathworks (2009): MATLAB - Mathworks. - MATLAB \& Simulink. http://doi.org/2016-11-26.

[19] May, Z., Amaran, M. H. (2011): Automated oil palm fruit grading system using artificial intelligence. - International Journal of Video and Image Processing and Network Security IJVIPNS-IJENS 11(3): 30-35.

[20] Nandi, C. S., Tudu, B., Koley, C. (2012): An automated machine vision based system for fruit sorting and grading. - Proceedings of the $6^{\text {th }}$ International Conference on Sensing Technology, ICST, 18-21 Dec, Kolkata, India.

[21] Nandi, C. S., Tudu, B., Koley, C. (2016): A machine vision technique for grading of harvested mangoes based on maturity and quality. - IEEE Sensors Journal 16(16): 63876396.

[22] Onur, C. (1988): Nar. - Derim. Narenciye Araş. Enst. Antalya, Özel Say1 5(4): 47.

[23] Papageorgiou, E. I., Aggelopoulou, K. D., Gemtos, T. A., Nanos, G. D. (2013): Yield prediction in apples using fuzzy cognitive map learning approach. - Computers and Electronics in Agriculture. http://doi.org/10.1016/j.compag.2012.11.008.

[24] Prasad, G. N. R., Vinaya Babu, D. A. (2006): A study on various expert systems in agriculture. - Georgian Electronic Scientific Journal: Computer Science and Telecommunications 4(11): 81-86.

[25] Qureshi, W. S., Payne, A., Walsh, K. B., Linker, R., Cohen, O., Dailey, M. N. (2017): Machine vision for counting fruit on mango tree canopies. - Precision Agriculture. http://doi.org/10.1007/s11119-016-9458-5.

[26] Razak, T. R., Mahmod, O., Bakar, M. N. B., Mansor, R. (2012): Mango grading by using fuzzy image analysis. - International Conference on Agricultural, Environment and Biological Sciences, May 26-27, Phuket, Thailand.

[27] Sahebkar, A., Ferri, C., Giorgini, P., Bo, S., Nachtigal, P., Grassi, D. (2017): Effects of pomegranate juice on blood pressure: A systematic review and meta-analysis of randomized controlled trials. - Pharmacological Research. http://doi.org/10.1016/j.phrs.2016.11.018.

[28] Spiegel, M. R., Schiller, J. J., Srinivasan, R. A. (2009): Schaum's Outlines of Probability and Statistics. - McGraw-Hill Education, New York.

[29] Srinivasan, S. P., Malliga, P., Nirmalraj, J. (2009): A fuzzy logic based decision support system to forecast the yield of Jatropha in cultivable wastelands. - IE and EM 2009. Proceedings 16th International Conference on Industrial Engineering and Engineering Management, Beijing, China, 21-23 October.

[30] Suksawat, B., Komkum, P. (2015): Pineapple quality grading using image processing and fuzzy logic based on Thai Agriculture Standards. - Proceedings 2015 International Conference on Control, Automation and Robotics, ICCAR, May 20-22, Singapore.

[31] Teoh, Y. K., Abu Hasan, S., Sauddin Saduddin, S. (2013): Automated mango fruit grading system using fuzzy logic. - J. Agric. Sci. DOI: 10.5539/jas.v6n1p41.

[32] Wang, X., Ouyang, Y., Liu, J., Zhu, M., Zhao, G., Bao, W., Hu, F. B. (2014): Fruit and vegetable consumption and mortality from all causes, cardiovascular disease, and cancer: systematic review and dose-response meta-analysis of prospective cohort studies. - BMJ. http://doi.org/10.1136/bmj.g4490. 
[33] Ying, L. C., Arbaiy, N., Salikon, M. Z. M., Rahman, H. A. (2017): Plant watering management system using fuzzy logic approach in oil palm nursery. - Journal of Telecommunication, Electronic and Computer Engineering 9(3-7): 129-134.

[34] Zadeh, L. A. (1983): A computational approach to fuzzy quantifiers in natural languages. - Computers \&Mathematics with Applications 9: 149-184.

[35] Zimmermann, H. J. (2001): Fuzzy Set Theory and Its Applications. - Kluwer Academic Publishers, London. http://doi.org/10.1007/978-94-010-0646-0. 